\title{
LA RESPONSABILIDAD SOCIAL EN LA EMPRESA FAMILIAR
}

\author{
M. A. HERNÁNDEZ-CALZADA ${ }^{*}$, J. MENDOZA-MOHENO, B. C. SALAZAR-HERNÁNDEZ \\ Universidad Autónoma del Estado de Hidalgo \\ martin_hernandez@hotmail.com* \\ Recebido 06/07/2016 - Aceptó 18/09/2017 \\ DOI: $10.15628 /$ holos.2016.4805
}

\section{RESUMEN}

El objetivo del presente artículo consiste en determinar la importância que tiene la responsabilidade social en las empresas familiares, analizando las motivaciones que llevan a los empresários a realizar acciones de responsabilidade social, así como la forma en que las empresas familiares conciben a la responsabilidad social. El análisis permite observar que las empresas familiares suelen llevar a cabo en mayor medida acciones de responsabilidade social que aquellas cuyo control, gestión y propiedad no está en manos de la familia. Así mismo, se vislumbra la necesidad de diferenciar la filantropia con la responsabildad social, así como la necesidad de generar en los empresarios un mayor conocimiento, tanto de lo que es, como de lo que implica la responsabilidad social.

PALAVRAS-CHAVE: Responsabilidad social, empresa familiar, motivativaciones

\section{SOCIAL RESPONSIBILITY IN THE FAMILY COMPANY}

\begin{abstract}
The aim of this research is to highlight the importance that social responsability has in the Family owned businesses, analyzing the motivations that the owners have in taking actions of social responsability, as well as the way Family owned businesses conceive social responability. The analysis allows to observe that Family owned businesses take ina more degree actions of social
\end{abstract}

responsability, than those enterprises whose control, management and property is not in the hands of families. Also, the need to differ philantropy with social responsability is perceived, as well as the need of generating in the owners a better understanding of what is, as well as what social responsability implies.

KEYWORDS: Social responsibility, family owned business, motivations 


\section{INTRODUCCIÓN}

La responsabilidad social parece ser un tema de moda, ha sido investigada desde diferentes facetas y sigue siendo un aspecto que permanece pendiente en las agendas de las organizaciones y los gobiernos.

La responsabilidad social tiene su origen desde la década de los '70, pero es a partir de los escándalos financieros vividos en los ' 90 , derivado de los malos manejos y corrupción de las empresas, cuando cobra importancia.

Por su parte, las empresas familiares han llamado la atención en discusiones privadas y públicas, debido al desarrollo económico y social que tiene este tipo de empresas, al ser una forma tradicional de hacer negocios (Mandl, 2008), con características propias que las distingue de otras cuya propiedad, gestión y control no están en manos de familias. En relación con la responsabilidad social, algunas investigaciones señalan que las empresas familiares tienen una mayor conciencia y son más activas en la sociedad, comparado con las empresas no familiares (Wiklund, 2006; Cennamo, Berrone, Cruz y Gómez-Mejía, 2012). A partir de ello, nuestra motivación se centra en llevar a cabo una investigación documental con el fin de constatar si las empresas familiares efectivamente son más proclives a la responsabilidad social y qué tipo de acciones llevan a cabo.

El objetivo del presente documento consiste en llevar a cabo una revisión de la literatura con el fin de determinar la evidencia de las empresas familiares como negocios comprometidos con la responsabilidad social empresarial, así como las motivaciones que las impulsa a realizar acciones de responsabilidad social. El documento está organizado en tres partes. En la primera parte se discuten las diferentes conceptualizaciones propuestas por los investigadores de la responsabilidad social. En la segunda parte se presentan las principales motivaciones que llevan a los empresarios a realizar acciones de responsabilidad social. El tercer apartado profundiza en la situación que guardan las empresas familiares en acciones de responsabilidad social. Finalmente se exponen las conclusiones a las que se arribó con la investigación.

\section{REVISIÓN DE LA LITERATURA}

\subsection{Definiendo el concepto de responsabilidad social empresarial}

La responsabilidad social en las empresas ha sido denominada indistintamente como responsabilidad social empresarial y como responsabilidad social corporativa, sin embargo, Delgado (2012) sugiere la utilización del término Responsabilidad Social de las Empresas, ya que el término de Responsabilidad Social Corporativa se relaciona generalmente con las empresas de gran tamaño, por lo que en esta investigación utilizaremos el término de Responsabilidad Social Empresarial (RSE) que engloba al total de las empresas, sin importar su tamaño.

En sus inicios, la responsabilidad social recibió críticas, como la que hace Friedman (1970), quien considera que la única responsabilidad social que deben tener las empresas, es la de incrementar sus utilidades por medios legales, sin ninguna otra obligación para con la sociedad. En la actualidad, el concepto de RSE, involucra acciones económicas, sociales y ambientales que deben llevar a cabo las empresas para el beneficio social. En este sentido, Ríos, López y López 
(2015) enfatizan que la RSE es una filosofia empresarial que enfatiza la necesidad de implementar nuevas formas de dirigir las empresas, a través de una gestión responsable con los stakeholders o grupos de interés.

Es importante señalar como lo destaca Sánchez (2012), el concepto de responsabilidad social analizado por vários autores, debe ser considerado como un fenómeno de gestión empresarial que debe orientarse no sólo a la rentabilidade de la empresa en sí, sino a satisfacer a las partes interesadas al interior de la empresa y en su entorno, que sea desarrollada de manera voluntaria y que vaya más allá de la legislación y las normas.

En latinoamérica, especificamente en México, la Alianza por la Responsabilidad Social Empresarial en México (AliaRSE) del Centro Mexicano para la Filantropía (CEMEFI), define la responsabilidad social empresarial como el compromiso consciente y congruente de cumplir integralmente con la finalidad de la empresa, tanto en lo interno, como en lo externo, considerando las expectativas económicas, sociales y ambientales de todos sus participantes, demostrando respeto por la gente, los valores éticos, la comunidad y el medio ambiente, contribuyendo así a la construcción del bien común (Cajiga, s/f). La RSE se refiere al conjunto de actividades voluntarias de la empresa que demuestran la inclusión de preocupaciones sociales y ambientales en las operaciones del negocio y las interacciones con los grupos de interés (Aragón e Iturrioz, 2013).

La responsabilidad social ha sido definida bajo diversas perspectivas. De la Garza y Guzmán (2013) advierten que la RSE es un concepto heterogéneo que depende de la percepción de la sociedad y de la propia organización. En este sentido, Arredondo (2013) enfatiza que en Europa la RSE se concibe de manera diferente, destacando más a los empleados y a la comunidad, en lugar de los accionistas, además la RSE parte de los empleados hacia las actividades de la compañía y no de la compañía hacia los empleados. En México, la responsabilidad social es entendida de la empresa hacia sus stakeholders como son los empleados, clientes, proveedores, gobierno, etc.

Hay que destacar que en ocasiones el término de responsabilidad social suele confundirse con la filantropía, sin embargo, es necesario distinguir, tal como lo señalan Contreras, López y López (2012), que la filantropía se limita a la aportación de donaciones a organizaciones de beneficiencia pública, mientras que la responsabilidad social es un concepto más amplio que involucra el beneficio común de todos los grupos de interés con los que la empresa tiene vínculos.

Existen ambigüedades en los resultados que relacionan la RSE y la competitividad (Quintana, 2008). Por un lado, algunos autores defienden que cuando las empresas implementan acciones de RSE en las actividades cotidianas, obtienen beneficios en el ámbito financiero, comercial, laboral, legal y medio ambiental, lo cual genera grandes ventajas a la organización (Contreras, López y López, 2012; De Souza, Soares, Pasa y Farache, 2010), sin embargo otros autores ponen en duda el beneficio en ventajas competitivas.

\subsection{Motivaciones para la responsabilidad social}

Las empresas tienen diferentes motivaciones para el involucramiento en acciones de responsabilidad social. Quazi y O'Brien (2000) en su investigación, afirman que el compromiso hacia la responsabilidad social depende de la visión del empresario y los resultados que de ella se 
espera obtener. En este sentido, la visión puede ser reducida o amplia. La primera se limita a la obtención de beneficios bajo un marco legal, es decir, las empresas son responsables socialmente cuando la obtención de utilidades y otros beneficios incluyen a la legalidad como único requisito a cumplir, coincidiendo con lo planteado por Friedman (1970). En la segunda, el empresario busca satisfacer expectativas de la sociedad, en términos medioambientales, conservación de recursos y filantropía.

Las motivaciones entonces pueden ser de tipo extrínseco, basadas en aspectos financieros, o en motivos intrínsecos como la percepción de RSE como una tarea moral (Graafland, Kaptein y Mazereeuw, 2010), relacionados con le ética del empresario. Por su parte, Murillo y Lozano (2006) clasifican la motivación del empresario al involucramiento de acciones de responsabilidad social, en motivaciones ideológicas y morales, y por otro lado, razones pragmáticas que resultan de la presión por parte de los grupos de interés.

En otra investigación llevada a cabo por Campopiano, De Massis y Cassia (2012), las motivaciones de los empresarios son clasificadas en económicas y éticas. Dentro de las motivaciones económicas están aquellas empresas que incrementan sus utilidades, para mejorar su reputación, llevando a cabo acciones tales como mejoras en condiciones de vivienda y en educación, así como incrementar los niveles de seguridad y las condiciones de trabajo de los empleados, la satisfacción de las necesidades de los consumidores y apoyar al gobierno en caso de desastres naturales. Dentro de las motivaciones económicas también están incluidas las acciones que buscan recuperar la imagen, previamente dañada. Campbell (2007) señala que cuando la reputación de una empresa está comprometida, resulta difícil continuar haciendo negocios con los consumidores y proveedores, lo que obliga a la empresa a preocuparse por la responsabilidad social para recuperar su imagen.

Respecto a las motivaciones éticas, están aquellos empresarios que buscan contribuir al bienestar de la comunidad y los preocupados por el ambiente, tal como se presenta en la figura 1:

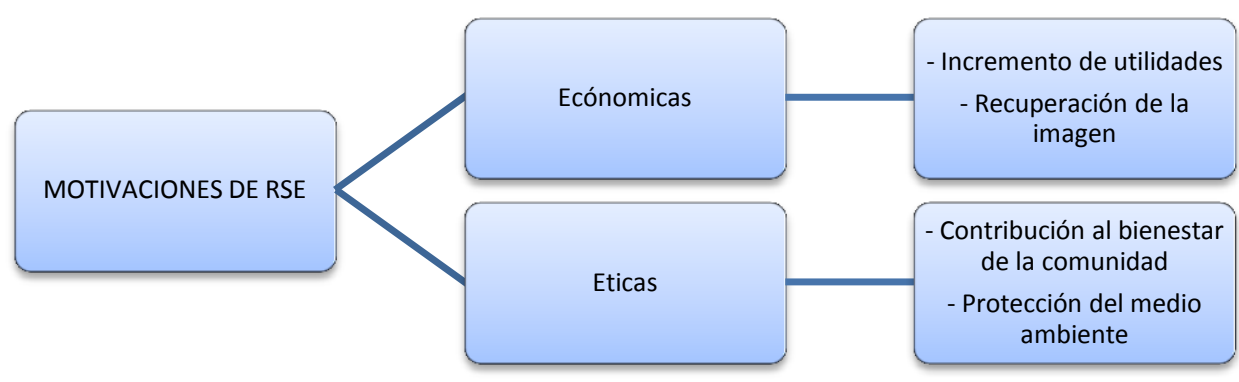

Figura 1: Motivaciones de los empresários

Fuente: Campopiano, De Massis y Cassia (2012)

Más allá de las motivaciones que los empresarios pueden tener en la RSE, para que las empresas lleven a cabo acciones de responsabilidad social, es fundamental la conciencia de ello. Quintana (2008) afirma la existencia de una falta considerable de conocimiento de RSE, lo que limita a las empresas a tomar acciones para ello. 
Contreras, López y López (2012) resaltan que entre las barreras que existen para el involucramiento en acciones de RSE están el capital cultural del empresario, donde propietarios con bajos niveles de escolaridad tienden a involucrarse en menor medida en acciones sociales. Connel (2007), por su parte, resalta como barreras significativas para un mayor compromiso con la RSE, la falta de tiempo y las restricciones financieras.

Según Cabrera, Déniz y Santana (2005), la responsabilidad social está relacionada con la naturaleza del tiempo, por un lado, están los que consideran que la RSE genera gastos a corto plazo, y en el otro extremo, están aquellos empresarios que evalúan los beneficios a largo plazo resultantes de la actuación social y perciben que las plusvalías superan en el tiempo los costos que se puedan derivar de la misma.

Existe una creencia generalizada que sostiene que la RSE es un compromiso que únicamente pueden asumir los grandes corporativos (Aldeanueva, 2012) y que las pequeñas empresas la visualizan más como un costo, que como una inversión (Ahmed, Montagno y Flenze, 1998), al estar más preocupados por su supervivencia, por lo que el tamaño de la empresa puede afectar al comportamiento de la misma (Sharma, 2004). El reporte llevado a cabo en el Reino Unido (Federation of Small Business, 2007), demuestra que los pequeños negocios también consideran importante el tema de RSE y perciben estar involucrados con ella. El $92 \%$ de las empresas pequeñas incluidas en la muestra de dicha investigación, considera que sus negocios son social y ambientalmente responsables. Los autores señalan que en ocasiones a los propietarios de pequeños negocios les es poco familiar el término de RSE y lo definen como buenas prácticas del negocio.

En una investigación llevada a cabo por Ríos, Ferrer y López (2013) en Celaya, Guanajuato, se demuestra que la RSE no es reconocida por las empresas familiares, especialmente por las de tamaño micro, que perciben que su actividad está primordialmente ligada al aspecto económico, sin que esté relacionado con actividades en beneficio de sus grupos de interés. La diferencia con las empresas del Reino Unido, citadas en el párrafo anterior, se puede deber a que la RSE es vista de manera diferente en Europa, debido a que destaca más a los empleados y a la comunidad, como grupos de interés de las empresas, en lugar de los accionistas (Arredondo, 2013), aunado a que los clientes y consumidores suelen ser más críticos a la hora de comprar productos o servicios, tomando en cuenta además de otros factores como la calidad y el precio, la responsabilidad social que tienen las empresas en cuestiones ecológicas, sociales y laborales, es decir, favorecen a aquellas empresas que en sus procesos no contaminan, que no tienen prácticas de explotación de empleados y que se preocupan por la sociedad, de lo contrario, los consumidores suelen castigar a las empresas, dejando de comprar y consumir sus productos y servicios.

Los pequeños negocios tienen un impacto en la economía local, son mayoritariamente propiedad de familias y ponen un especial énfasis en la reputación y calidad del producto/servicio (Besser y Jarnagin, 2007) y se preocupan más por involucrarse en los problemas de su localidad, tomando acciones concretas de RSE, por lo que la responsabilidad social en poblados pequeños puede tener un mayor impacto que en las grandes ciudades, donde la población ignora las acciones que las empresas llevan a cabo sobre responsabilidad social, lo que provoca un menor involucramiento de los propietarios en este tipo de acciones. 
Entre las principales acciones ambientales que los pequeños negocios llevan a cabo están (Connell, 2007):

a) Minimización de la basura y reciclaje

b) Compra de productos alternativos que son ambientalmente amigables

c) Medidas de eficiencia de energía

d) Reducción del uso del transporte por el staff

e) Cambio de productos/servicios

f) Reducción del uso de transporte para el negocio

g) Utilización de recursos de energías renovables

h) Inversión en tecnologías limpias

La RSE ha cobrado importancia en países como México. En una investigación llevada a cabo en Monterrey, México, Pérez (s/f), señala que los propietarios de grandes corporativos han mostrado su preocupación en relación con la vivienda de sus trabajadores, su salud y la educación de los empleados y sus hijos, siendo la fe religiosa la gran motivación. Así mismo, esta autora, después de llevar a cabo una investigación de las 25 empresas más importantes del país, según el informe de las "500 de Expansión", obtuvo que la responsabilidad social no aparece como una de las preocupaciones esenciales en más de la mitad de las empresas más importantes en México, ya que no se tiene un responsable de las acciones de responsabilidad social, sino que son gestionadas por el departamento de recursos humanos, mercadotecnia o comunicación. Las empresas llevan a cabo acciones de filantropía, sin embargo, únicamente una de las 25 empresas analizadas tiene una estrategia formal para la RSE. Las motivaciones de los empresarios mexicanos para llevar a cabo acciones de RSE están basadas en la coacción social y la presión del mercado, como una respuesta a los grupos de interés para mejorar su imagen, siendo por tanto una motivación esencialmente económica.

En la investigación llevada a cabo por Campopiano, De Massis y Cassia (2012) del análisis de 25 casos de enseñanza de grandes corporativos en el mundo, se encontró que, a excepción de Grupo Bimbo, junto con otras 2 empresas, los comportamientos de la responsabilidad social del resto, se orientan a motivaciones económicas para mejorar su reputación, o incluso su desempeño, ausentes de motivaciones morales y ética de los empresarios.

A pesar de que la RSE es un tema relativamente nuevo para los empresarios de países como México, la investigación llevada a cabo en Celaya por Contreras, López y López (2012), demuestra que los empresarios cada vez tienen mayor conocimiento del término de responsabilidad social empresarial y están conscientes de sus implicaciones, aunque sean de manera parcial, esporádica e informal.

\subsection{Empresa familiar y responsabilidad social empresarial}

Las empresas familiares son realidades multidimensionales por su naturaleza (Uhlaner, 2002) y tienen un comportamiento único derivado de las interrelaciones que se producen entre la familia y el sistema empresarial (Cabrera y Déniz, 2005). 
Los grupos de interés con los que está interrleacionada la empresa familiar influyen en forma decisiva en las acciones de RSE, ya que la satisfacción de las demandas de los grupos de interés es determinante para la propia supervivencia de la empresa. En este sentido, las empresas familiares pueden tener comportamientos positivos $\mathrm{y}$ negativos respecto a sus empleados, propietarios, clientes, la sociedad y otros grupos de interés (Cabrera, Déniz y Santana, 2005), donde la familia está en el centro de la compañía, situándose como el principal grupo de interés (Mandl, 2008).

Tradicionalmente, las empresas familiares tienen una alta participación en actividades filantrópicas, derivado del íntimo nexo que existe entre la familia y la empresa. En este tipo de empresas, el apellido de las familias está ligado al negocio, por lo que la imagen es sumamente cuidada. Cuando las empresas familiares tienen un comportamiento considerado como no deseado socialmente, la imagen de la familia se ve devaluada, lo que las obliga a actuar, no nada más bajo su propio interés, sino en beneficio de los empleados y la sociedad en general. Wiklund (2006) señala que en el caso de las empresas no familiares, los propietarios institucionales son anónimos, representados por los negocios que los contratan, mientras que en el caso de las empresas familiares, los propietarios y los miembros de la familia tienen su riqueza atada a la empresa y son fácilmente identificables y bien conocidos por la sociedad en general.

La reputación de la familia cobra una especial importancia, donde las decisiones tomadas y las acciones llevadas a cabo por la empresa deben ser vigiladas con especial atención para mantener la buena imagen, lo que motiva a los propietarios de las empresas familiares a ser más propensos a invertir recursos en RSE para construir y mantener una buena imagen (Dyer y Whetten, 2006) y elevar la reputación de la familia y su visibilidad con los clientes, proveedores y la sociedad (Campopiano, De Massis y Cassia, 2012). La imagen, por lo tanto, se convierte, de acuerdo con la Teoría de Recursos y Capacidades, en un recurso valioso, raro, no sustituible e inimitable, que constituye la base de la ventaja competitiva (Cabrera, Déniz y Santana, 2005).

Las empresas familiares comparten metas y valores y se caracterizan por su alta motivación, cohesividad y compromiso con la fuerza de trabajo (Campopiano, De Massis y Cassia, 2012); sus trayectorias de crecimiento son estables y continuas comparadas con las de las empresas no familiares, y toman rutas más dinámicas y volátiles. En contraparte, se pueden presentar el paternalismo y el nepotismo, así como la existencia de decisiones emocionales e informales, debido al dominio de la gestión de la familia (Mandl, 2008). Sin embargo, la orientación a largo plazo, aunada a la gran adaptación al entorno (Aldeanueva, 2012), influyen de forma positiva en las acciones de RSE llevadas a cabo por la empresa.

Debido al personalismo y el deseo de sostener la visión a través de las distintas generaciones, y retener el control de la familia, las empresas familiares se comportan en una forma particular, substituyendo los objetivos de riqueza económica y racional por objetivos que ayudan a acumular riqueza socio-emocional, reputación e imagen de la empresa (Sharma y Sharma, 2011), lo que permite la consistencia de los miembros familiares a través de las diferentes generaciones en las iniciativas de RSE y los valores que las inspiran son transferidos de una a otra generación (Campopiano, De Massis y Cassia, 2012).

Mandl (2008) confirma lo anterior argumentando que cuando una empresa es transferida a la siguiente generación, no sólo los recursos financieros son transferidos, sino también el capital social y cultural, observándose la sostenibilidad en el largo plazo a través del cambio 
generacional, existiendo compromiso e involucramiento de los miembros de la familia con la empresa y las actividades de RSE.

Quintana (2008) manifiesta la existencia de una relación directa entre el buen gobierno de la empresa familiar y la RSE, siendo el consejo de familia y el consejo de administración fundamentales en las decisiones, sin embargo, la voluntad de la familia es imprescindible a la hora de tomar acciones relacionadas con la RSE. Este autor sostiene que la familia, el consejo de administración y la dirección aportan eficiencia al sistema, ya que permiten mejorar las relaciones con el resto de los grupos de interés (proveedores, clientes, acreedores, empleados). Este tipo de decisiones son tomadas sobre todo por el Consejo de Familia, órgano de gobierno y dirección, donde la familia está involucrada para tomar las decisiones en torno a la empresa.

En este sentido, las empresas familiares diseminan en mayor medida las acciones de RSE, a través de reportes de responsabilidad social, códigos de ética, páginas web, reportes medioambientales, y políticas de sustentabilidad (Campopiano y De Massis, 2015). A través de la comunicación de la RSE, los grupos de interés, tales como proveedores y clientes, toman decisiones de negocios y de compra hacia empresas preocupadas por la sociedad.

De acuerdo con el Instituto de Empresa Familiar (2009), las acciones sociales suelen confundirse con la RSE, siendo necesario distinguir lo que son actividades aisladas fuera de un plan, y la RSE que debe ser parte de la estrategia del negocio. En países como México, existe participación de las empresas familiares en actividades de RSE como son la asistencia social, las actividades religiosas, las actividades festivas y las actividades laborales (Ríos, Ferrer y López, 2013).

Así mismo, en una investigación llevada a cabo en el estado de Aguascalientes, México, los resultados muestran que, si bien las empresas familiares de tamaño micro y pequeño tienen una conducta propicia al uso voluntario de medidas ambientales y de salud, la realidad es que la adopción de programas voluntarios como la norma ISO 14001 o el Certificado de Industria Limpia es casi inexistente (González, Leal y Bogar, 2013). En otra investigación llevada a cabo en la ciudad de Celaya, GUanajuato, las empresas familiares contemplan la posibilidad de crecimiento con la posible injerencia en la generación de empleos para personas fuera del núcleo familiar (Contreras, López y López, 2012).

Factores tales como el tipo y grado de implicación de la familia determinan de igual forma la RSE. En la investigación llevada a cabo por Sharma y Sharma (2011), el involucramiento de la familia influye en el negocio en las actitudes, las normas subjetivas y el control de comportamiento percibido, que determinan la extensión para llevar a cabo una estrategia ambiental proactiva. Las empresas familiares con menores niveles de conflicto dentro de la familia controladora son más exitosas en traducir las intenciones dominantes de coalición para colocar recursos para la consecución de la estrategia ambiental proactiva.

A partir de lo anterior, los propietarios de empresas familiares tienen diversas motivaciones para llevar a cabo acciones de responsabilidad social, desde motivaciones económicas, como morales. Un factor importante a considerar es el nivel cultural, no sólo del empresario, sino de la sociedad en general. La sociedad otorga una gran importancia a las organizaciones comprometidas en reportar sus actividades y principios socialmente responsables (Fisher, Gunz y McCutcheon, 2001). En países desarrollados, la sociedad está involucrada en mayor medida en este tipo de acciones y sus decisiones de compra se basan, no sólo en la 
valoración de la relación calidad-precio, sino en la imagen y reputación de la empresa, basado en el involucramiento con sus stakeholders. En México, las decisiones de compra se basan principalmente en el poder adquisitivo del consumidor, donde aún cuando tuviera conocimiento de abusos hacia proveedores y empleados por parte de la empresa, son variables que no afectan em su decisión de compra.

Por otro lado, una de las principales barreras que limitan la decisión de responsabilidad social de las empresas familiares mexicanas, se basa en el tiempo, donde la valoración de los beneficios a corto plazo son valorados en mayor medida que los que pudieran alcanzarse en el largo plazo. Sin embargo, a pesar de ello, de acuerdo con los hallazgos y evidencia existente en la literatura, las empresas familiares tienden a preocuparse en mayor medida en acciones de responsabilidad social, que como lo hacen las empresas no familiares.

\section{CONCLUSIONES}

Aun cuando el concepto de responsabilidad social ha evolucionado con el tiempo, aún queda un largo camino por recorrer para que las empresas elijan la responsabilidad social como acto moral y filosofía del negocio, sin buscar únicamente el beneficio económico, incluida la mejora de la imagen. Por lo tanto, la visión de responsabilidad social de los empresarios sigue siendo reducida, según la clasificación que proponen Quazi y O’Brien (2000), siendo necesario avanzar hacia una visión amplia, basada no únicamente en el cumplimiento de regulaciones legales y búsqueda del incremento de utilidades, sino como una convicción y satisfacción de tipo morales.

La responsabilidad social está relacionada con aquellas organizaciones comprometidas con la sociedad. En el caso de las empresas familiares se observa una aparente mayor responsabilidad social, derivado de la presión que tienen los propietarios por parte de los grupos de interés, aunado al nexo directo existente entre el apellido de las familias y el propio negocio, ya que un comportamiento negativo de la empresa daña la imagen de la familia.

Las empresas familiares suelen ser filantrópicas y suelen llevar a cabo acciones sociales, sin embargo, no siempre son empresas socialmente responsables, ya que las acciones distan de estar integradas a las políticas y a la estrategia del negocio.

El tamaño de la empresa y la percepción del tiempo son variables que determinan la RSE. Comúnmente se tiene la idea de que únicamente los grandes corporativos son quienes llevan a cabo acciones de RSE, sin embargo, los micro y pequeños empresarios llevan a cabo acciones de responsabilidad social, pero la confunden con buenas prácticas del negocio. El horizonte de tiempo de la RSE debe ser a largo plazo, puesto que únicamente a lo largo del tiempo se pueden contabilizar los beneficios económicos y competitivos, de lo contrario, únicamente se concibe como un alto costo.

Una de las principales limitantes que existe en la RSE es el bajo nivel de conocimiento que se tiene del concepto, siendo necesaria una mayor difusión para concientizar a los propietarios, labor que deben llevar a cabo, tanto el gobierno, los organismos reconocidos como la CEMEFI en México y los investigadores del tema.

A pesar de que los grandes corporativos tienen mejores prácticas de RSE que las empresas de menor tamaño, en los pequeños poblados, los propietarios de empresas tienen un mayor 
involucramiento, en actividades sociales y laborales, debido a la relación socioemocional tan cercana con la población.

En general, las empresas llevan a cabo acciones de responsabilidad social motivadas por razones de tipo económico, según la clasificación de motivos que guían a las empresas familiares a tomar acciones de RSE propuesta por Campopiano, De Massis, y Cassia (2012), sin embargo, no se puede generalizar, pues puede haber empresas familiares que hacen intentos $y$ sus motivaciones están basadas en la ética y la moral.

La verdadera RSE debe ser integral, es decir, debe incluir acciones sociales, laborales y ambientales, sin descuidar ninguna de ellas, siendo parte de la estrategia del negocio, lo que exige que debe ser planeada, controlada y gestionada.

\section{REFERENCIAS}

AHMED, N.U., MONTAGNO, R.V., FLENZE, R.J. Organizational performance and environmental consciousness: An empirical study. Management Decision, V. 36, n. 36, p. 57-62, 1998.

ALDEANUEVA, I. La responsabilidad social corporativa en la empresa familiar: el enfoque de los grupos de interés. Gestión Joven Revista de Agrupación Joven Iberoamerciana de Contabilidad y Administración de Empresas, V. 9, p. 40-51, 2012

ARAGÓN, C., ITURRIOZLANDART, C. Family owner's and social responsibility in small and médium family firms. Documento obtenido el 3 de agosto de 2014 desde http://www.ebenspain.org/docs/Papeles/XXI/family-owner-s-and-social-responsibility-in-small-and-mediumfamily-firms.pdf., 2013.

ARREDONDO, F.G. La responsabilidad social de las empresas transnacionales españolas en México en el marco del TLCUEM. En F.S. Leal Medina y J.F. Ojeda Hidalgo (comp.), Desarrollo de la responsabilidad social en las organizaciones p. 179-206, México: UAA, 2013.

BESSER, T. L., JARNAGIN, S.K. Corporate social responsibility: Small business and small towns. USA: Center for ethical business, 2010.

CABRERA, M.K., DÉNIZ, M.C. Corporate social responsibility and family business in Spain.Journal of Business Ethics, V. 56, n. 1, p. 27-41, 2005.

CABRERA, M.K., DÉNIZ, M.C., SANTANA M, D.J. Responsabilidad social corporativa y empresa familiar. Revista Europea de Dirección y Economía de la Empresa, V. 14, n.4, p. 43-58, 2005.

CAJIGA, J.F. El concepto de responsabilidad social empresarial. México: Centro Mexicano para la Filantropía. http://www.cemefi.org/esr/images/stories/pdf/esr/concepto_esr.pdf

CAMPBELL, J.L. Why would corporations behave in socially responsible ways? An institutional theory of corporate social responsibility.Academy of Management Review, V. 4, p. 497-505, 2007.

CAMPOPIANO, G., DE MASSIS, A., CASSIA, L. The relationship between motivations and actions in corporate social responsibility: An exploratory study. International Journal of Business ans Society, V. 13, n. 3, p. 391-425, 2012.

CAMPOPIANO, G., DE MASSIS, A. Corporate social responsibility reporting: A content analysis in 
family and non-family firms. Journal of Business Ethics, V. 129, p. 511-534, 2015.

CENNAMO, C., BERRONE, P., CRUZ, C., GÓMEZ, L.R. Socioemotional wealth and proactive stakeholder engagement: Why family controlled firms care more about their stakeholders. Entrepreneurship Theory \& Practice, V. 36, n. 6, p. 1153-1173, 2012.

CONNELL, N. Social and environmental responsibility and the small business owner. UK: The Federation of Small Businesses, 2007.

CARROLL, N. Social and environmental responsibility and the small business owner.Federation of Small Businesses, 2007.

CONTRERAS, R., LÓPEZ, A., LÓPEZ, C. El altruismo de la empresa familiar y la posible cultura de la responsabilidad social de las empresas. Administración y Organización, p. 102-119, dic. 2012.

DE LA GARZA, M.T., GUZMÁN, E. Estrategia organizacional en la responsabilidad social corporativa: una opción competitiva para las organizaciones. En F.S. LEAL., J.F. OJEDA (comp.), Desarrollo de la responsabilidad social en las organizaciones (p. 79-94). México: UAA, 2013.

DELGADO, D. La responsabilidad social corporativa: una apuesta por el compromiso cívico de las empresas. Madrid: Fundación Ciudadanía y Valores, 2012.

DYER, W.G., Jr., WHETTEN, D.A. Family firms and social responsibility: Preiliminary evidence from the S\&P 500. Entrepreneurship Theory and Practice, V .30, p. 785-802, 2006.

FEDERATION OF SMALL BUSINESS. Social and environmental responsibility and the small business owner. UK: FSB, 2007.

FISHER, J., GUNZ, S., MCCUTCHEON. Private/public interest and the enforcement of a code of professional conduct. Journal of Business Ethics, V. 31, n. 3, p. 191-207, 2001.

FRIEDMAN, M. The social responsibility of business is to increase its profits. New York Times Magazine, Sept. 13, 1970.

GONZÁLEZ, R., LEAL, F.J., GARCÍA, B. Los pequeños empresarios en Aguascalientes y el uso voluntario de medidas de protección ambiental en la empresa. En .S. LEAL, J.F. OJEDA (comp.), Desarrollo de la responsabilidad social en las organizaciones (149-160). México: UAA, 2013.

GRAAFLAND, J., VAN DE VEN, B. Strategic and moral motivation for corporate social responsibility. Journal of Corporate Citizenship, V. 22, p. 111-123, 2006.

Instituto de la Empresa Familiar. La responsabilidad social empresarial en la gran empresa familiar española. Madrid: IEF, 2009.

MANDL, I. Overview of family business relevant issues. Vienna: KMU Forschung Austria, 2006.

MURILLO, D. y LOZANO, J.M. SME'S and CSR: An approach to CSR in their own words. Journal of Business Ethics, V. 67, n. 3, p. 227-240, 2006.

PÉREZ. La responsabilidad social corporativa en México: ¿ser o parecer? Análisis de la comunicación en 25 empresas del país (s/f). Obtenido el 30 de junio de 2014 desde: http://www.reddircom.org/textos/marielaperez.pdf

QUAZI, A.M., O'BRIEN, D. An empirical test of a cross-national model of corporate social responsibility. Journal of Business Ethics, V. 25, p. 33-51, 2000. 
RÍOS, M., FERRER, J., LÓPEZ, A. Participación de las empresas familiares en actividades de responsabilidad social sostenible. Revista Panorama Empresarial, V. 7, n. 12, p. 35-46, 2013.

RÍOS M., LÓPEZ A., LÓPEZ M. Ética y calidad laboral: impacto en el desempeño empresarial. Un estudio empírico. Holos, V. 31, n. 3, p. 308-320, 2015.

QUINTANA, J. Responsabilidad social en las empresas familiares. Madrid: Foretica.

SÁNCHEZ, M.D. Responsabilidad Social Empresarial: Gobernanza Corporativa, Empresa y ONG. Oñati Socio-Legal Series, Vol. 2, No. 3, P. 139-159, 2012

SHARMA, P. Stakeholder mapping technique: Towards the development of a family firm typology., hace 2004. IFERA Fourth Conference, Jönköping, 2008.

SHARMA, P., SHARMA, S. Drivers of proactive environmental strategy in family firms.Business Ethics Quarterly, V. 21, n. 2, p. 309-334, 2011.

UHLANER, L.M., BERENT-BRAUN, M.M., JEURISSEN, R., WIT, G. Beyond size: Predicting engagement in environmental practices of Dutch SMEs. Journal of Business Ethics, V. 109, n. 4, p. 411-429, 2012.

WIKLUND, J. Commentary: Family firms and social responsibility: Preliminary Evidence from the S\&P 500. Entrepreneurship Theory and Practice, November, p. 803-808, 2006. 